\title{
Studies of age-related changes in blood perfusion coherence using wearable blood perfusion sensor system
}

Yulia I. Loktionova, Evgeny A. Zherebtsov, Elena V. Zharkikh, Igor O. Kozlov, Angelina I. Zherebtsova, et al.

Yulia I. Loktionova, Evgeny A. Zherebtsov, Elena V. Zharkikh, Igor O. Kozlov, Angelina I. Zherebtsova, Victor V. Sidorov, Sergei G. Sokolovski, llya E. Rafailov, Andrey V. Dunaev, Edik U. Rafailov, "Studies of age-related changes in blood perfusion coherence using wearable blood perfusion sensor system," Proc. SPIE 11075, Novel Biophotonics Techniques and Applications V, 1107507 (22 July 2019); doi: 10.1117/12.2526967 


\title{
Studies of age-related changes in blood perfusion coherence using wearable blood perfusion sensor system
}

\author{
Yulia I. Loktionova ${ }^{\mathrm{a}}$, Evgeny A. Zherebtsov, ${ }^{\mathrm{a}, \mathrm{b}}$, Elena V. Zharkikh ${ }^{\mathrm{a}}$, Igor O. Kozlov ${ }^{\mathrm{a}}$, \\ Angelina I. Zherebtsova ${ }^{a}$, Victor V. Sidorov ${ }^{c}$, Sergei G. Sokolovskie, Ilya E. Rafailov ${ }^{\text {, }}$ \\ Andrey V. Dunaev ${ }^{a}$, and Edik U. Rafailovd, e

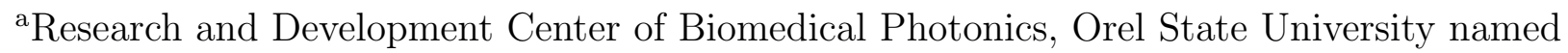 \\ after I.S. Turgenev, Orel, Russia \\ ${ }^{\mathrm{b}}$ Optoelectronics and Measurement Techniques, University of Oulu, Oulu, FI-90014, Finland \\ "SPE "LAZMA" Ltd, Moscow, Russia \\ dAston Medical Technology Ltd., Birmingham, UK \\ eAston Institute of Photonic Technologies, Aston University, Birmingham, UK
}

\begin{abstract}
Laser Doppler flowmetry (LDF) was used for detection of age-related changes in the blood microcirculation. The LDF signal was simultaneously recorded from the 3rd fingers' pads of both hands. Amplitudes of the blood flow oscillations and wavelet coherence of the signals were used for the data analysis. A statistical difference in the synchronisation of myogenic oscillations was found between the two studied age groups. Myogenic oscillations of blood perfusion in the younger group had a higher wavelet coherence parameter than in the older group. Observed site-specific and age-related differences in blood perfusion can be used in the future in the design of experimental studies of the blood microcirculation system in patients with different pathologies.
\end{abstract}

Keywords: laser Doppler flowmetry, microcirculation, blood flow oscillations, wavelet coherence, ageing

\section{INTRODUCTION}

The cardiovascular system is one of the most important systems of the human body. With age it is exposed to various trophic disorders, resulting in blood microcirculation disturbances which can adversely affect the course of diseases and the development of concomitant complications. ${ }^{1}$ Therefore, for conducting a reliable diagnosis, it is necessary to formulate the concept of the normal state of blood microcirculation for different age groups.

One of the most common non-invasive optical methods for measuring blood perfusion is laser Doppler flowmetry (LDF). The method is based on laser sensing of tissue and further analysis of scattered light, which is reflected from erythrocytes. LDF is used for functional diagnostics of the blood microcirculation system, including diagnostics of socially significant diseases associated with the cardiovascular system, as well as their complications. ${ }^{2-4}$ Furthermore, this method allows for the evaluation of the oscillatory processes in the microcirculatory bed. There are several frequency ranges that characterise the contribution of various factors to the LDF signal: endothelial $(0.0095-0.021 \mathrm{~Hz})$, neurogenic $(0.021-0.052 \mathrm{~Hz})$, myogenic $(0.052-0.145 \mathrm{~Hz})$, respiratory $(0.145-0.6 \mathrm{~Hz})$ and cardiac $(0.6-2 \mathrm{~Hz}) .^{5}$

LDF studies are conducted not only at rest, but also with a variety of functional samples, such as local occlusion tests or heating. ${ }^{6} \mathrm{LDF}$ is also used to study age-related changes in blood flow. ${ }^{7}$ But currently, there is a shortage of studies about the simultaneous detection of the blood perfusion signal from symmetrical areas of upper limbs. ${ }^{8,9}$

The purpose of this work was to investigate the synchronisation of blood perfusion signals from contralateral limbs in two different age groups.

\footnotetext{
Send correspondence to Yulia Loktionova

E-mail: julya-loktionova@mail.ru
}

Novel Biophotonics Techniques and Applications V, edited by Arjen Amelink, Seemantini K. Nadkarni, Proc. of SPIE-OSA Vol. 11075, 1107507 - @ 2019 SPIE-OSA · CCC code: 1605-7422/19/\$21 · doi: 10.1117/12.2526967 


\section{MATERIALS AND METHODS}

Experimental studies were conducted using two wearable laser Doppler flowmetry monitors "AMT-LAZMA 1" (Aston Medical Technology Ltd., UK) for the analysis of blood microcirculation. The devices implement identical channels for recording blood perfusion and allow simultaneous measurements at several points of the body. At the moment, these devices are the latest development to assess the state of blood microcirculation. ${ }^{10}$

These studies were conducted in accordance with the principles set out in the Helsinki Declaration of 2013 by the world medical Association. The studies were approved by the Ethics committee of Orel State University. The study involved 40 healthy volunteers without cardiovascular and other serious chronic diseases that affect the blood circulation system; volunteers with alcohol or drug dependence were also excluded. Before starting the study, each volunteer gave a voluntary informed written consent to participate in the experiment and passed a questionnaire to identify possible health problems. The study participants were divided into two groups according to their age: 22 volunteers were under the age of 20 (1st group: average age of $19.4 \pm 0.6$ years), 18 volunteers were over 40 (2nd group: average age of $52.6 \pm 10.2$ years).

Studies were conducted in a sitting position, in a state of physical and mental rest, not earlier than 2 hours after eating. The volunteer's hands were placed on the table at the heart's level. The index of blood microcirculation was recorded for 10 minutes, while the sensors were attached to the palmar surface of the distal phalanx of 3rd fingers without applying any pressure on the study area.

These sites were chosen because they represent the glabrous skin that covers the face, palms and soles of the feet. The mechanism of sympathetic regulation dominates at these locations due to the presence of arteriovenous anastomoses. ${ }^{11}$

\section{RESULTS AND DISCUSSION}

Volunteers were recruited in such a way that the second group was much older than the first $(p<0.05)$. In other parameters (heart rate and blood pressure) no significant difference was observed.

The study revealed a higher level of perfusion in group 2 compared to group 1 . Statistically significant differences between the two groups were found in amplitudes of amplitudes of endothelial $(A e)$ and myogenic $(A m)$ oscillations. These results are presented in Table 1.

Table 1. Results of the wavelet analysis of the obtained data

\begin{tabular}{|l|l|l|l|l|l|l|}
\hline Parameters & Im, PU & Ae, PU & An, PU & Am, PU & Ar, PU & Ac, PU \\
\hline Younger group & $14.4 \pm 4.7 *$ & $0.74 \pm 0.32 *$ & $0.35 \pm 0.15$ & $0.56 \pm 0.26 *$ & $0.61 \pm 0.30$ & $0.59 \pm 0.28$ \\
\hline Older group & $18.2 \pm 3.0$ & $0.59 \pm 0.56$ & $0.35 \pm 0.16$ & $0.43 \pm 0.23$ & $0.55 \pm 0.35$ & $0.63 \pm 0.42$ \\
\hline
\end{tabular}

* - The significance of the difference between the values was confirmed with $p<0.05$ using Mann-Whitney test

Fig.1 shows the results of comparisons of the wavelet coherence parameters on the fingertip for two groups of subjects. The box-and-whiskers diagram was built for the demonstration of differences in coherence parameters between healthy volunteers of the selected age groups. Myogenic oscillations of blood perfusion in the younger group had a higher wavelet coherence parameter than in the older group, according to Wilcoxon signed-rank test.

Wavelet coherence shows how much the blood flow oscillations coincide in frequency and amplitude. In this case, the magnitude of the amplitudes is not taken into account, the value is the ratio of the amplitudes of the oscillations. The closer the amplitudes are to each other, the higher the coherence (approaching 1), and the closer the similar amplitudes are in the frequency range, the higher the coherence.

Fluctuations in blood flow at a frequency of approximately $0.1 \mathrm{~Hz}$ (the so-called vasomotions) characterise the microvascular activity of smooth muscle cells. It was shown in the previous studies that the prevalence of vasomotions in humans is significantly dependent on the pathology and experimental conditions. Such pathological conditions as diabetes, hypertension, and peripheral arterial occlusive disease (PAOD) are associated with changes in the pattern of vasomotion. ${ }^{12}$ Since the subjects included in both of the studied groups were healthy 


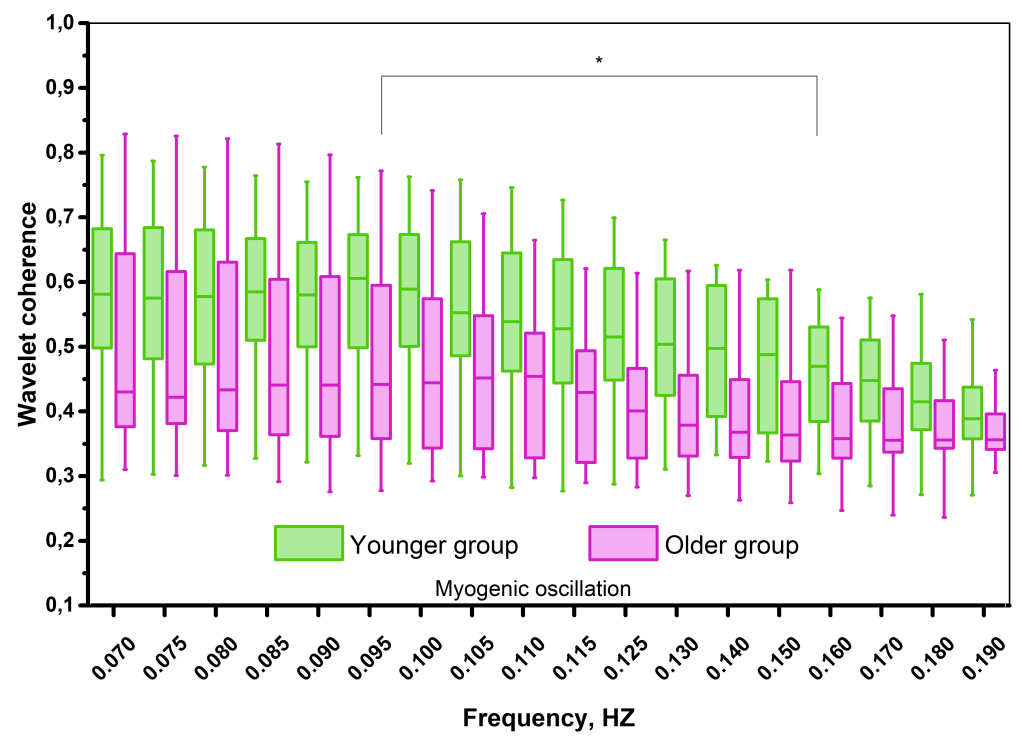

Figure 1. Analysis of wavelet coherence of blood perfusion between the middle fingers of the right and left hands of 1st (green boxes) and 2nd (purple boxes) groups of volunteers.

* - The significance of the difference between the values was confirmed with $p<0.05$ according to the Wilcoxon signed-rank test

volunteers, without identified health problems and had similar blood pressure and pulse parameters, we assume that the result obtained is associated with the age specificity of the microvasculature.

It can be said that with age the work of mechanisms that synchronise oscillations in this frequency interval throughout the cardiovascular system is changing, causing differences in the vascular tone of the right and left parts of the body. The obtained results are consistent with the data obtained earlier by other researchers, confirming that oscillations of around $0.1 \mathrm{~Hz}$ are highly synchronised in the cardiovascular system of young healthy subjects. ${ }^{9}$

\section{CONCLUSIONS}

The blood microcirculation system is subjected to significant structural and functional changes during aging. The presented study has shown differences in the wavelet coherence parameters of two distinct age groups. It was discovered that myogenic oscillations of blood perfusion in the younger group had a higher wavelet coherence parameter than in the older group.

Also, it is necessary to continue research with an increase in the samples number and size. The use of wavelet analysis of the received LDF signals to detect changes in the microcirculation regulatory mechanisms during ageing is also promising. The obtained data can be considered further in the development of protocols for the studies of the blood microcirculation system in patients with different pathologies.

\section{ACKNOWLEDGMENTS}

This study was funded by the Russian Science Foundation (the research project 18-79-00237). Evgeny Zherebtsov kindly acknowledges for personal support from grant of Academy of Finland No.318281 (data processing). 


\section{REFERENCES}

[1] Makrantonaki, E. and Zouboulis, C., "Characteristics and pathomechanisms of endogenously aged skin," Dermatology 214(4), 352-360 (2007).

[2] Zherebtsova, A. I., Zherebtsov, E. A., Dunaev, A. V., Podmasteryev, K. V., Pilipenko, O. V., Krupatkin, A. I., Khakhicheva, L. S., and Muradyan, V. F., "Study of the functional state of peripheral vessels in fingers of rheumatological patients by means of laser doppler flowmetry and cutaneous thermometry measurements," Proc. SPIE 9917, 99170M (2016).

[3] Dremin, V. V., Filina, M. A., Zharkikh, E. V., Kozlov, I. O., Zherebtsov, E. A., Potapova, E. V., Malaya, N. S., Yakushkina, N. Y., Snimshchikova, I. A., and Dunaev, A. V., "Peculiarities of local blood microcirculation in patients with psoriasis," Proc. SPIE 10685, 1068532 (2018).

[4] Zharkikh, E. V., Dremin, V. V., Filina, M. A., Makovik, I. N., Potapova, E. V., Zherebtsov, E. A., Zherebtsova, A. I., and Dunaev, A. V., "Application of optical non-invasive methods to diagnose the state of the lower limb tissues in patients with diabetes mellitus," Journal of Physics: Conference Series 929(1), 012069 (2017).

[5] Lancaster, G., Stefanovska, A., Pesce, M., Marco Vezzoni, G., Loggini, B., Pingitore, R., Ghiara, F., Barachini, P., Cervadoro, G., Romanelli, M., and Rossi, M., "Dynamic markers based on blood perfusion fluctuations for selecting skin melanocytic lesions for biopsy," Scientific Reports 5, 12825 (2015).

[6] Tikhonova, I. V., Tankanag, A. V., and Chemeris, N. K., "Timeamplitude analysis of skin blood flow oscillations during the post-occlusive reactive hyperemia in human," Microvascular Research 80(1), 58-64 (2010).

[7] Li, L., Mac-Mary, S., Marsaut, D., Sainthillier, J. M., Nouveau, S., Gharbi, T., de Lacharriere, O., and Humbert, P., "Age-related changes in skin topography and microcirculation," Archives of Dermatological Research 297(9), 412-416 (2006).

[8] Bernjak, A., Stefanovska, A., Urbancic-Rovan, V., and Azman-Juvan, K., "Quantitative assessment of oscillatory components in blood circulation: classification of the effect of aging, diabetes, and acute myocardial infarction," Proc. SPIE 5692, 5692-11 (2005).

[9] Tankanag, A. V., Grinevich, A. A., Kirilina, T. V., Krasnikov, G. V., Piskunova, G. M., and Chemeris, N. K., "Wavelet phase coherence analysis of the skin blood flow oscillations in human," Microvascular Research 95, 53-59 (2014).

[10] Zherebtsov, E., Sokolovski, S., Sidorov, V., Rafailov, I., Dunaev, A., and Rafailov, E. U., "Novel wearable VCSEL-based blood perfusion sensor," Proceedings - International Conference Laser Optics 2018, ICLO 8435409, 564 (2018).

[11] Urbancic-Rovan, V., Stefanovska, A., Bernjak, A., Azman-Juvan, K., and Kocijancic, A., "Skin blood flow in the upper and lower extremities of diabetic patients with and without autonomic neuropathy," Journal of Vascular Research 41(6), 535-545 (2004).

[12] Aalkjær, C., Boedtkjer, D., and Matchkov, V., "Vasomotion - what is currently thought?," Acta Physiologica 202(3), 253-269 (2011). 